\title{
Impact of Wall Coating on the Behavior of Indoor OWC under Diffuse Topology
}

\author{
Mohamed B. El-Mashade \& Hanaa H. Qamer \\ Electrical Engineering Dept., Faculty of Engineering, Al Azhar University, Nasr City, Cairo, Egypt \\ Email: mohamed.b.elmashade@azhar.edu.eg, hanna.helmy@gmail.com
}

Received: 23 November 2020; Accepted: 10 January 2021; Published: 08 February 2021

\begin{abstract}
Optical wireless communication (OWC) is an innovative technology that is gaining more attention as the demand for capacity continues to increase. It is one of the most promising alternative technologies for indoor and outdoor applications. In this paper, the effect of the inner wall coating material, color and roughness on the performance of OWC system implementing single-diffuse topology is studied. A new procedure is proposed to generate a rough surface model with predetermined statistical properties to simulate the matte painting material on the internal walls of a room. Additionally, a new technique that applies the geometrical theory of diffraction (GTD) in conjunction with a ray tracing (RT) scenario is developed to evaluate the scattered optical beam due to a primary ray incident on a Lambertian surface. The performance of the single-diffuse OWC strategy is assessed by investigating some important performance measurements such as signal strength and the bit error rate (BER) due to unavoidable ambient light which is modeled as an additive white Gaussian noise. It is shown that the surface roughness of the matte painting on the Lambertian diffuse surface has a major effect on the indoor OWC system performance.
\end{abstract}

Index Terms: Indoor OWC, inner wall coating material, single-diffuse topology, GTD-RT procedure, Lambertian surface, rough surface, color and roughness effects.

\section{Introduction}

Communication and information technologies have been regarded as the key constituents of social and economic development owing to their ability to create new benefits and conveniences that were not experienced before. Indeed, communication enters our daily lives in so many different ways that is very easy to overlook the multitude of its facets. In this regard, modern communication relies deeply on wireless technology. The radio frequency spectrum is considered as the backbone of this strategy of exchanging information. Day-by-day, the wireless technology is speedily developing. Actually, the allocation policy of the radio spectrum leans on assigning the communication channels to specific consumers with licenses for specific wireless technologies and services, which means that the available spectrum is statically divided into licensed and unlicensed frequency bands. While the use of the first type is restricted to authorized operators, the bands of the second category are available for use by the public, subject only to transmission constraints [1].

The measurements around the world have revealed that wide ranges of the spectrum are rarely used most of the time, while other bands are heavily occupied. Depending on the location, time of the day, and frequency bands, the spectrum is actually found to be underutilized. However, those unused portions of the spectrum are licensed and thus cannot be used by systems other than the license owners. Hence, there is a need for a novel technology that can benefit from these opportunities. The dynamic management of the allowable spectrum represents the best strategy to its issue of scarcity. This strategy depends upon sharing unoccupied channels with unlicensed users without interfering with the signals of the licensed consumers. This is the main goal of cognitive radio (CR) which is the most recommended technique for the problem of spectrum scarcity $[2,3]$.

Since an abundance of wireless techniques have been developed, and continuously increased capacity is being requested, a severe problem has emerged. With emerging technologies and with the increasing number of wireless devices, the radio spectrum is becoming increasingly congested.

As the calling of increasing the capacity of communications is unavoidable circumstance, the optical spectrum can serve as a good spectrum resource for wideband wireless communications. In other words, optical wireless communication (OWC) is regarded as one of the most favorable technologies for applications in indoor as well as outdoor situations. This style of communications constitutes a potential solution for replying the ever-increasing demand for increased wireless capacity in the conventional radio frequency (RF) band. In other words, OWC can play a significant role as a complementary technology to the RF systems in future information superhighways. In this regard, it can 
provide flexible networking solutions that exhibit cost-effective, highly secure high-speed license-free wireless broadband connectivity for a lot of applications, including enterprise connectivity, video and entertainment, voice and data, disaster recovery, illumination and data communications, along with surveillance [4-6].

Typically, the mechanism of diffuse composes of a transmitter that is directly guided towards the ceiling emitting a wide optical beam. Since it doesn't require neither careful alignment of the transmitter and receiver modules, nor a lineof-sight (LOS) path to be maintained along with its almost immunity to transmission path blockage, the diffuse OWC indoor topology represents the most appropriate procedure for both infrastructure and LAN adhoc networks. However, diffuse links experience high path loss for a horizontal separation of several meters. The path loss is increased further if a temporary impediment conceals the receiver in such a way that the main signal path is blocked. This situation is referred to as shadowing. Additionally, a photo-detector with a wide field of view (FOV) is normally required to collect signals that have undergone one or more reflections from ceiling, walls and room objects. Such reflections make the signal more attenuated. Moreover, the received signal may suffer from severe multipath dispersion, where the transmitted pulses spread out in time over alternative routes of differing lengths, which in turn results in limiting the maximum un-equalized bit rate. Because of these factors, a relatively high optical transmitted power is requested. On the other hand, the average optical power emitted by an IR transceiver is constrained by eye safety regulations along with electrical power consumption in portable devices Thus, in diffuse systems, the use of power-efficient modulation mechanisms is desirable. Additionally, the entire room of operation needs to be illuminated by a single or multiple transmitter. This is naturally accomplished by diffused light propagation after a few reflections but demanding a relatively huge transmitted optical power [7-10].

Inner wall coating should meet some common requirements such as desired colors, glossy or matte surface, good appearance and thermal comfort. Actually, the inner wall coatings have significant effects on the indoor OWC implementing diffuse topology. On the other hand, heat is normally associating with the infrared radiation incident on or emitted from an object. Off course, this is applicable for infrared radiation falling on the inner walls of a room. The wavelengths of the infrared radiation producing heat ranging from 700 to $1100 \mathrm{~nm}$. This range of the infrared spectrum is commonly used in indoor OWC systems. The signal strength and, consequently, the performance parameters affecting the indoor wireless optical links depend strongly on the infrared reflectance of the inner walls of buildings. Here, the reflectance means the fraction of the incident optical power that is reflected from the wall surface [11].

Besides their visual properties, the paints on the roofs, outer or inner walls of the building may be prepared to cause thermal comfort and energy savings [12]. During winter climate, on one hand, it is usually required to warm up the inner rooms of the building. Paints with high reflectance in the thermal infrared region of the spectrum will cause most of the incident infrared energy to be reflected back to the room area keeping the room warm. The summer climate, on the other hand, needs low-reflectance paint materials on the walls of the rooms. Such types of paints absorb most of the incident infrared energy emitted by sources inside the room and thereby they leak the heat energy to the outside via heat conduction. This may help in keeping the room cool. Consequently, the inner wall paints are developed to have suitable level of reflectance in the thermal infrared region. The reflectance of such wall covers depends on the material, color and roughness of the coating surface. This reflectance is a function of the wavelength of the radiation that is incident on the walls [13-16]. As a consequence of this wavelength dependence, a dispersion and/or distortion of the received signal may be occurred especially when multipath phenomenon is encountered during indoor communications. As the indoor OWC systems commonly operate in the infrared band, the impact of the wall paint material on the performance of the OWC systems is of importance to be studied. Up to the author's knowledge, rare literature has shown interest in such a study. Therefore, the goal of the present research is to perform complete quantitative investigations about the influence of the commercially available wall paints on the practically operating OWC systems. The rest of the manuscript is organized as follows. Section 2 is concerned with literature review in the field of the paper. The basic assumptions of our developing mechanisms are outlined in section 3. Section 4 discusses the characteristics of the optical wave scattering on the inner wall surface. Performance of OWC systems is evaluated in section 5. Section 6 is devoted to our simulation as well as experimental results. Finally, our concluded remarks are summarized in section 7.

\section{Literature Review}

An experimental free-space infrared link, of $50 \mathrm{Mb} / \mathrm{s}$, for indoor OWC has been established in [17]. In that experiment, an eye-safe $806 \mathrm{~nm}$ transmitter, directed at the ceiling, emits an on-off-keyed (OOK) signal having $475 \mathrm{~mW}$ average power. The receiver employs a $1.0 \mathrm{~cm}^{2} \mathrm{Si}$ p-i-n photodiode coupled to a high-index hemispherical concentrator, which is covered by an optical bandpass filter of $30 \mathrm{~nm}$ bandwidth. A decision-feedback equalizer mitigates the effect of multipath inter symbol interference (ISI). In the presence of bright skylight, the link achieves a range of $2.9 \mathrm{~m}$ at $10^{-7}$ bit error rate (BER).

In [18], the non-directed indoor channels, that use IM/DD of an infrared carrier at $\lambda=832 \mathrm{~nm}$ have been experimentally characterized. The frequency responses of these channels were measured over the range of $2-300 \mathrm{MHz}$ and the obtained results were used to compute the channel impulse responses, path losses and root means square (RMS) delay spreads. It was found that un-shadowed LOS configurations have smaller path losses, RMS delay spreads and 
power penalties than their corresponding ones in diffuse counterparts. Shadowed LOS configurations, on the other hand, exhibited larger values for all the three parameters than the corresponding shadowed diffuse configurations.

The performance of an indoor optical wireless spot-diffusing system is theoretically assessed under the impact of multipath dispersion and ambient light noise [19]. For the diversity-detection case, a receiver comprising an array of narrow FOV detectors oriented in different directions was used to maximize the collected signals and minimize noise. A novel line-strip multi-beam system (LSMS) is investigated with single and diversity receiver configurations. It was concluded that the performance improvement, obtained through the use of LSMS with a three-direction diversity receiver, is of about $20 \mathrm{~dB}$ signal-to-noise ratio (SNR) enhancement over the conventional diffuse system.

Actually, since the indoor OWC systems commonly operate in the infrared band, the impact of the wall paint material on the performance of OWC systems is of great importance to be assessed. Up to the author's knowledge, rare literature has shown interest in this field of research. This prompts the necessity to investigate such study. This ability to study the impact of wall paint material on the performance of OWC strategy is the major contribution of this work.

\section{Research Assumptions}

A new robust computational strategy, that applies the geometrical theory of diffraction (GTD) in conjunction with the ray tracing (RT) method, is developed to evaluate the electromagnetic scattering pattern that is generated by an incident plane wave on a rough surface of quite arbitrary statistical parameters. Experimentally, a primary laser beam is assumed to be incident on the room's ceil, which is supposed to be Lambertian rough surface. The Fresnel reflection model is applied, under the assumption of arbitrary electrical and optical properties of the rough surface material, to obtain the scattering patterns for both the power reflected to the upper half-space and the power transmitted to the medium covered by the rough surface. Additionally, the polarization of the plane wave is taken into account and the correlation function is assumed to be Gaussian for an isotropic rough surface. The developed algorithm accounts for multiple bounces of an incident ray. Hence, it can be considered as an arbitrary higher-order GTD-RT procedure. The numerical results of the present work are concerned with investigating the dependence of the scattering pattern on the surface roughness, refractive index, angle of incidence, and the resolution of the geometric model of the rough surface.

\section{Optical Wave Scattering Evaluation on the Inner Wall Surface}

The painting material on the inner walls of the buildings may be glossy, semi-glossy or matte. Thus, the surfaces of such coated walls are considered as random rough surfaces with roughness degree that greatly affects the optical scattering when subjected to an incident optical beam. The scattering of the optical power incident on a rough surface can be classified as one of two types: specular reflection and diffuse reflection. In the case of specular reflection, all the rays making specific angle of incidence with the surface are reflected at the same angle, whereas in the diffuse reflection case, the scattered light has a wide range of directions constituting a broad beam. Examples of the specular and diffuse reflections are those caused by glossy and matte paints, respectively. Matte painting materials have mainly diffuse reflection, whilst glossy painting ones have specular as well as diffuse reflections. In comparison with other types of links, the optical diffuse communication links are less affected by shadowing and provide more flexibility as well as availability to mobile units. In contrast to point-to-point OWC, diffuse OWC do not require a LOS.
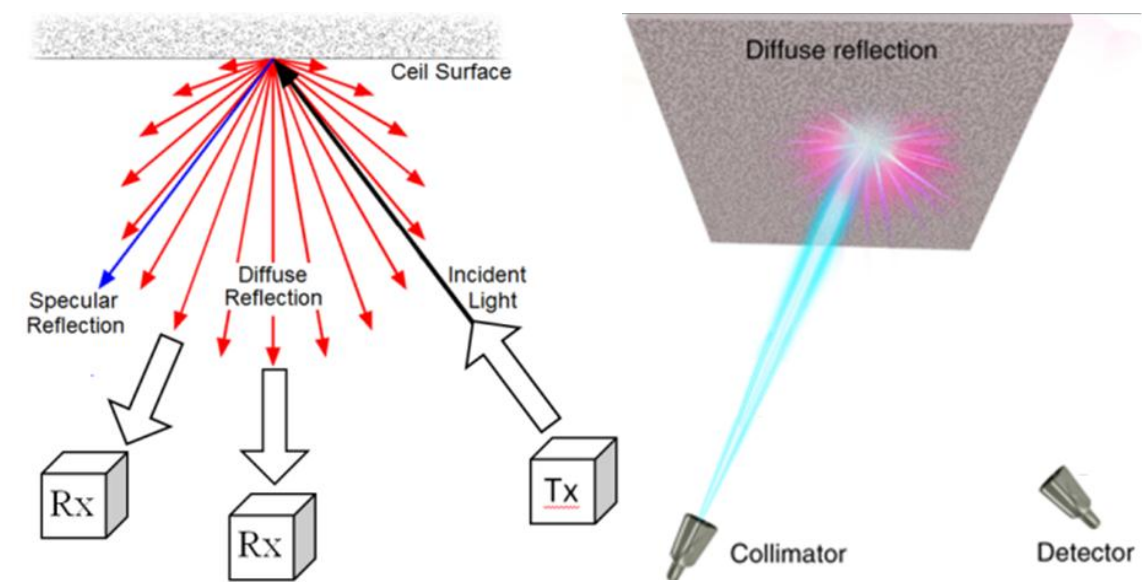

Fig.1. Diffuse reflection in indoor OWC system

To receive the diffuse reflections from the surrounding walls and the three-dimensional bodies existing in the room, the transmitters should be of large beam widths and the receivers must be of large FOVs as Fig.(1) demonstrates. As a consequence of these constraints along with the phenomenon of path diversity, the diffuse links are unaffected by 
obstacles and miss-alignment errors. However, the received signal is affected by multipath dispersion due to multiple reflections. Examples of surfaces resulting in efficient diffuse reflection of light are those made of non-absorbing powder such as plaster, those made from fibers such as paper, or those made from a polycrystalline material such as white marble. However, many types of painting materials cause both of specular and diffuse reflections simultaneously; the pattern of the beam scattered from surfaces of such types of materials seems like that presented in Fig.(2).

An ideal "matte" or diffusely reflecting surface has Lambertian reflectance property. In this regard, Lambertian surface has the same apparent brightness to any observer regardless of the observer's angle of view. In other words, the Lambertian surface's luminance is isotropic, and the luminous intensity obeys Lambert's cosine law. To evaluate the optical scattering from random rough surfaces, with arbitrary statistical parameters, the GTD method in conjunction with RT methodology will be used to assess the optical scattering that is resulting from an incident plane wave on the rough in arbitrary direction.

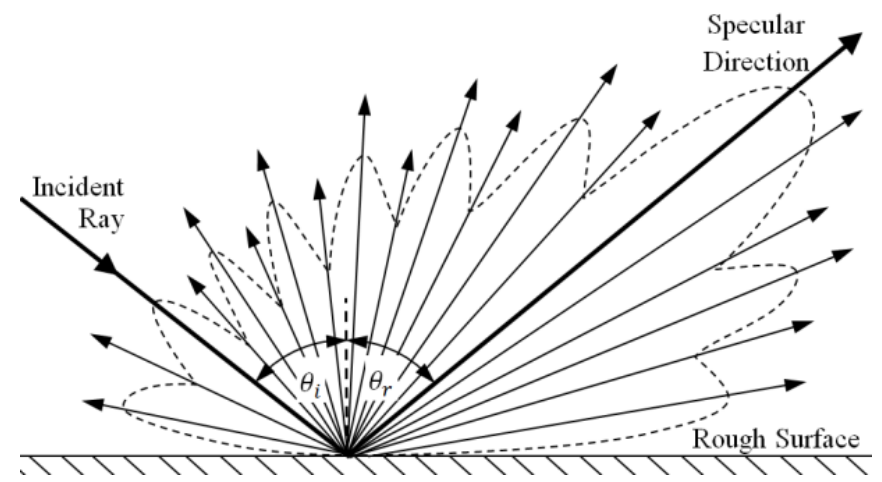

Fig.2. Scattering of an incident ray on a rough surface of high degree of roughness

The numerical assessment of the optical scattering on the inner surface of the walls requires the implementation of a geometric model to represent the random surface made by the painting layer on such walls of a room. It is proposed to use the spatial-domain procedure introduced in [13] to generate a random rough surface with arbitrary statistical properties. The most important ones of these properties of such type of random surfaces are the mean value of the surface height $\left(h_{\text {mean }}\right)$, the root-mean-squared-height $\left(h_{r m s}\right)$, and the correlation length between the heights of the neighboring points on the surface $\left(\mathrm{L}_{\mathrm{c}}\right)$. Owing to the well-known statistical characteristics of Gaussian surfaces, a great deal of research has been conducted to treat such type of rough surfaces. From the statistical point of view, Gaussian rough surfaces are completely defined by the correlation length $\mathrm{L}_{\mathrm{c}}$ and the root-mean-squared height $\mathrm{h}_{\mathrm{rms}}$. In the present analysis, it is assumed that, for isotropic rough surface, the correlation function is of the form of Gaussian and can be expressed as:

$$
C(d)=h_{r m s}^{2} \exp \left(-\frac{d^{2}}{L_{c}^{2}}\right)
$$

Where d denotes the horizontal distance between two correlated points on the rough surface. The slope of the surface can be calculated as the second moment of the power spectrum. A rough surface with Gaussian correlation function is known to have a slope of $s=\sqrt{2} h_{r m s} / L_{c}$ and the roughness of which is defined as:

$$
R_{D}=\triangleq \sqrt{2} s=2 \frac{h_{r m s}}{L_{c}}
$$

For an optically rough surface model representing a matte wall painting surface, the following conditions must be practically satisfied:

$$
h_{\text {mean }}=0, \lambda \ll h_{r m s} \& \lambda \ll L_{c}
$$

The geometric model of the random rough surface is discretized to a number of interconnected vertices in such a way that the distance between any connected vertices is not greater than $\Delta$. To get accurate numerical assessment of the optical wave scattering on such a surface using GTD with RT evaluation algorithm, the condition of $\Delta<<\lambda$ must be verified. If the previous conditions are fulfilled, the GTD-RT procedure can be applied to evaluate the scattering of an optical ray (or beam of rays) incident on such rough surface model and, hence, the diffuse pattern of light can be obtained. In order to achieve such evaluation resulting from an application of a plane wave illuminating a rough surface, the incident plane wave is represented by a number of parallel rays; each ray is associated with an amount of power that is to be calculated, so as a uniform power distribution over the transverse plane of the incident wave is established. The 
phase of each one of the scattered rays can be evaluated by computing its total distance travelled during ray tracing. On the other hand, the rough surface absorption or reflectance can be assessed by calculating the Fresnel coefficients at the point of incidence. Finally, an algorithm is proposed to construct the scattering pattern over the upper half space. Both first and higher orders of ray tracing are described through systematic scenario of form that can be easily applied as a simulation tool for optical and electromagnetic scattering evaluation on rough surface.

\section{Performance Assessment of OWC System}

The behavior of the diffuse OWC system can be assessed by investigating some important performance measures that metric its reaction against different operating conditions. Signal strength and bit error rate (BER) are the two interesting parameters that translate this reaction. For calculating the BER, the signal-to-noise ratio (SNR) should be firstly computed. It is assumed that the unavoidable ambient light interfering with the signal carried by the diffused optical beam is modeled as an additive white Gaussian noise (AWGN) with power spectral density $\mathrm{N}_{0} / 2(\mathrm{~W} / \mathrm{Hz})$. By assuming that the optical line-width is $\mathrm{B}_{\mathrm{w}}(\mathrm{Hz})$, the noise power becomes:

$$
P_{N}=\frac{1}{2} N_{0} B_{\mathrm{w}}
$$

The angular distribution of the optical signal strength (power) due to the single diffuse mechanism, as was previously described, can be evaluated as:

$$
P_{S}\left(\theta_{m}, \phi_{n}\right)=P_{R x} S\left(\theta_{m}, \phi_{n}\right)
$$

Hence, the computation of the SNR takes the form:

$$
S N R=\frac{P_{S}}{P_{N}}=\frac{P_{R x} S\left(\theta_{m}, \phi_{n}\right)}{\frac{1}{2} N_{o} B_{W}}
$$

In terms of $\mathrm{E}_{\mathrm{b}} / \mathrm{N}_{0}$, the formulation of the SNR tends to:

$$
S N R=\frac{P_{S}}{P_{N}}=\frac{E_{b} R_{b}}{\frac{1}{2} N_{o} B_{W}}
$$

In $\mathrm{dB}$ scale, Eq. (7) has a new form as:

$$
\left(E_{b} / N_{o}\right)_{d B}=S N R_{d B}-\left(R_{b} / B_{W}\right)_{d B}-3
$$

The calculation of the BER is strongly dependent upon the modulation technique implemented in the OWC system. The Mary-PSK is used here to assess the underlined optical diffuse topology. A reference curve, that depicts the relation between the BER and the SNR for Mary-PSK, is generated to get the assessed value of the BER.

\section{Results and Discussions}

Paints prepared for high reflectivity in the infrared region of the electromagnetic spectrum may be made of inorganic complexes which reflect the infrared radiation and selectively reflect visible radiation. The reflectivity and absorptivity are dependent upon the paint material. An infrared-reflective paint may be colored and can be synthesized as mixtures of oxides, nitrates, acetates or metal oxide. In the present work, three types of wall paints are considered: the standard yellow, cool yellow, and cool brown as are introduced in [4].

A primary laser beam is assumed to be incident on the room's ceil, which is supposed to be Lambertian rough surface and, thereby, causing diffuse reflection of the incident beam. This results in almost uniform scattering of the optical power in different directions throughout the room space. Off course, such a matte surface roughness has the major effect on the scattered optical beam width. For the following investigations, the rough surface model of the room's ceil is assumed to have a correlation length of $L_{c}=2.4 \mathrm{~mm}$ and a roughness degree of $R_{D}=0.225$. The angle of incidence of the primary optical beam on the room's ceil affects the shape of the scattered optical beam and this in turn impacts the signal strength over the interior of the room. It should be noted that the primary beam is almost normal to the rough surface of the room's ceil $\left(\theta_{i}=1^{\circ}\right)$ and, hence, the scattered beam is omnidirectional. The room dimensions are: $\mathrm{L}_{\mathrm{x}}=6 \mathrm{~m}, \mathrm{~L}_{\mathrm{y}}=5 \mathrm{~m}$, and $\mathrm{L}_{\mathrm{z}}=3 \mathrm{~m}$. 


\subsection{Dependence of the Wall Coating Reflectance on the Wavelength}

For each type of the three considered paints that can be used to coat the inner walls, the reflectance is plotted, as a function of the wavelength, in Fig. (3). In this figure, it is shown that the near-infrared spectra have considerable differences in the variation of the reflectance as the paint material as well as color varies. For the infrared region of the spectrum defined by $1000<\lambda<1700 \mathrm{~nm}$, the cool brown paint is more reflective than the standard yellow. For the entire range of the examined wavelengths (infrared and visible), the cool yellow paint presents higher reflectance than both the standard yellow and the cool brown. Off course, such disperssive behavior of the reflectance has a great effect on the optical signal strength in indoor OW links. Therefore, the paint material and color can greatly change the performance of this type of OWC systems.

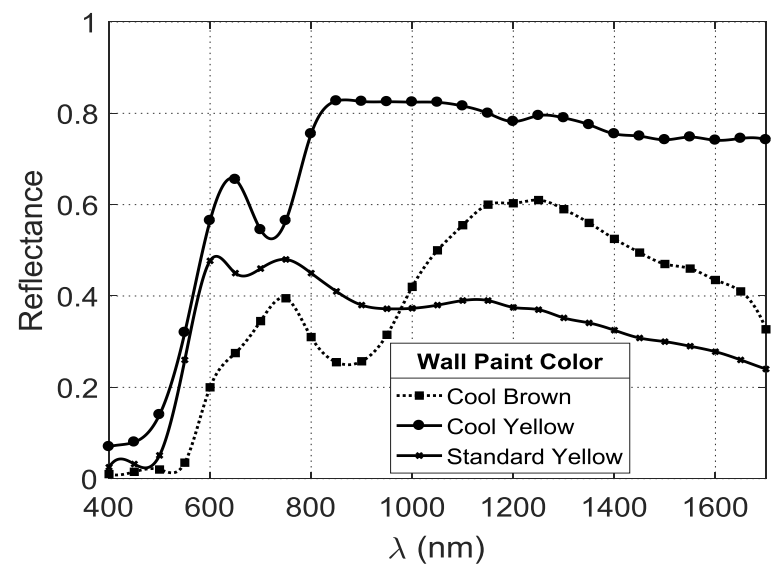

Fig.3. Reflectance dependence on wavelength of some cool and standard paint materials

\subsection{Experimental Assessment of Optical Scattering}

As previously mentioned, in OW links that employ diffuse topology, a primary optical beam of either visible or infrared radiation is subjected to diffuse reflection on the room walls. In this subsection, the effects of the material and color of wall coatings (thin sheets are used as alternatives for experimental assessment) on the scattering of an optical beam are experimentally investigated. Two experimental setups are arranged, as shown in Fig.(4), for the indicated purpose to be achieved. The first experimental setup is established to measure the radiation patterns of the visible and infrared emitting sources. The second practical setup uses these sources to measure the directional patterns of the optical scattering on sample of thin sheets identical to the coatings used in conventional painting of the room wall.

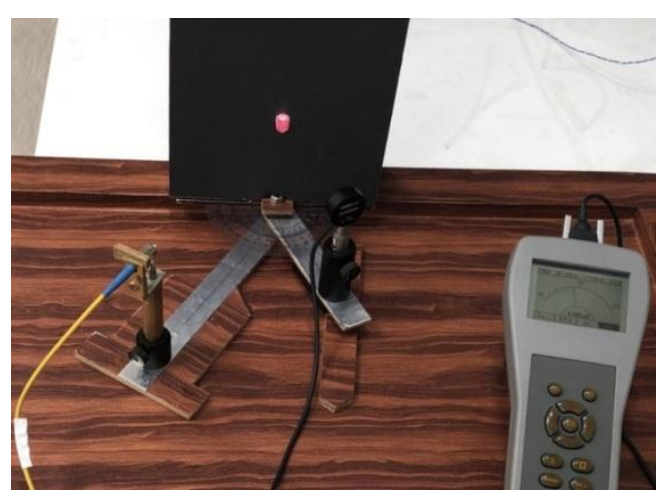

(a) Measurement of the LED beam

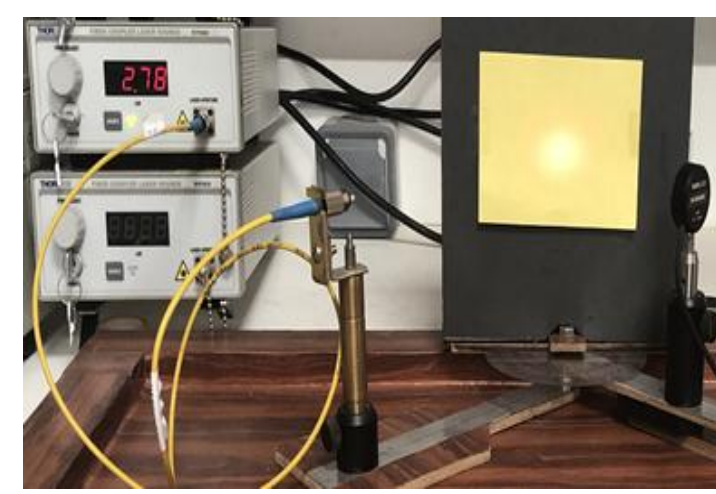

(b) Measurement of the optical scattering from the rough sheet

Fig.4. Experimental setup for measuring the beam of the light source and the scattering pattern excited by an optical beam incident on the rough sheet

In these experiments, the light source of the incident plane wave is of $\lambda=635 \mathrm{~nm}$. A rough sheet subjected to the incident beam is placed on a vertical wall. The light source is oriented in such a way that the incident beam makes an angle of $45^{\circ}$ with the normal to the sheet. An optical sensor connected to the power meter normally, with respect to the sheet, rotates from $-90^{\circ}$ to $90^{\circ}$ to read the intensity of the light scattered from the flat sheet under test. Two white sheets of different roughness degrees, a glossy white sheet of roughness $R_{D}=0.09$ and a matte white sheet of roughness, $\mathrm{R}_{\mathrm{D}}=0.225$, are employed for assessment of the proposed numerical procedure.

The radiation patterns of the light sources generating beams in infrared and visible regions of electromagnetic spectrum are displayed in Fig.(5). These patterns of radiation are measured by an optical power meter of model 
Thorlabs® PM100 with the optical sensor S120B. The half-power beam width (HPBW) of the visible light beam is about $10^{\circ}$, whereas that of the infrared beam is about $60^{\circ}$. The beams of these light sources are chosen for experimental measurements to obtain a variety of experimental results that can help in studying the effects that are encountered in the practical OW links employing diffuse topologies.

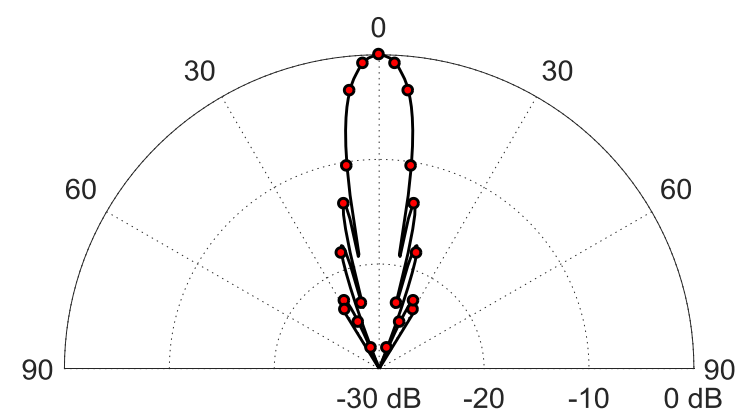

(a) Visible light beam $(\lambda=635 \mathrm{~nm})$

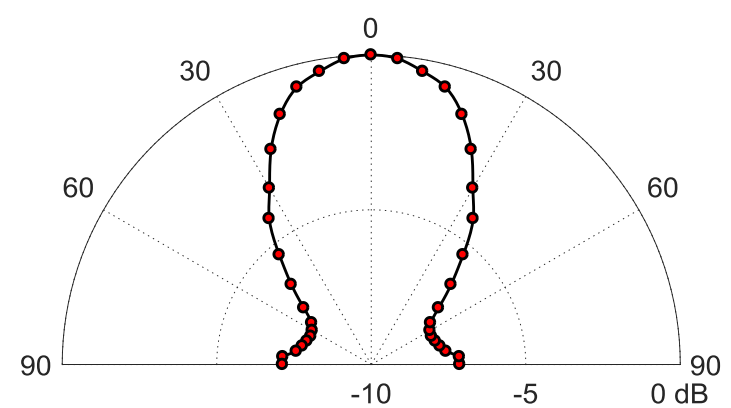

(b) Infrared beam $(\lambda=940 \mathrm{~nm})$

Fig.5. Experimental measurement of radiation patterns of the examined light sources

As a demonstrative experiment to show the effect of the surface roughness on the characteristics of the optical power scattering, the practical setup illustrated in Fig.(4-b) is used to measure the optical scattering from white sheets of different degrees of roughness. The measured directional pattern of the optical power scattering due to an incidence light beam of $\lambda=635 \mathrm{~nm}$ on a glossy white sheet of roughness $R_{D}=0.09$ and a matte white sheet of roughness $R_{D}=0.225$ are drawn in Fig.(6). It is shown that for smooth surfaces with relatively low degree of roughness, the scattering pattern includes both specular and diffuse features. On the other side, the directional pattern of scattering obtained from matte white sheet shows completely diffuse characteristics due to the relatively higher degree of roughness. However, the scattering in the specular direction is still slightly higher than the backscattering as the roughness degree is not enough to satisfy the Lambertian property of the rough surface.

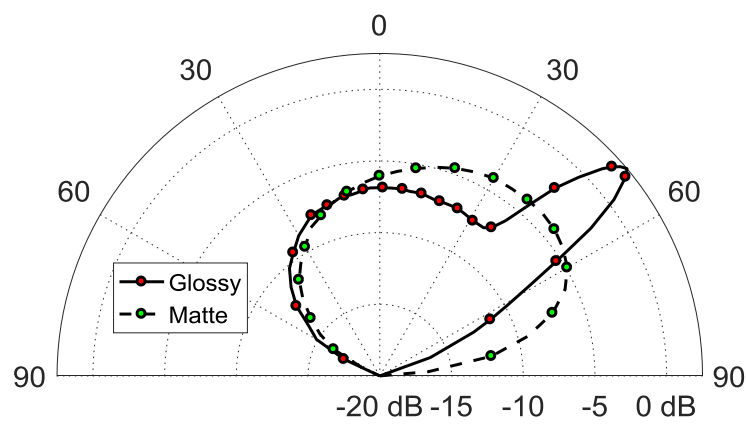

Fig.6. Measured scattering patterns of a visible light beam $(\lambda=635 \mathrm{~nm})$ incident at angle of $45^{\circ}$ on matte and glossy white sheets

In the next experimental work, three samples of colored matte sheets, with optical and thermal properties similar to those of the paint materials used for numerical investigations in the present work, are subjected to a normally incident infrared beam of $\lambda=940 \mathrm{~nm}$. The surface of each sheet is undergone the infrared beam in a manner similar to that applied in indoor OW links. The beam width is selected to illuminate a wide area on the reflecting walls so as to get good signal distribution over the room space. The measured directional patterns of the scattered optical power are displayed in Fig. (7). In this plot, it is noted that the cool yellow sheet has the maximum reflectance due to its infrared-reflective material along with its light color. In comparison with the cool yellow sheet, the standard yellow sheet has a considerably low reflectance as its material is not designed to highly reflect the infrared radiation. In spite of its material, which is designed to highly reflect the infrared radiation, the cool brown sheet has a considerably low reflectance, in comparison with the cool yellow sheet, which can be attributed to its darker color. Due to its material which is designed for high infrared reflectance, the cool brown sheet has higher level of infrared reflectance than that of the standard yellow sheet in spite of being of lighter color. This can be interpreted as that the impact of the infrared-reflective material of the cool brown sheet has a greater effect on the infrared reflectance than the lighter color of the standard yellow sheet. 


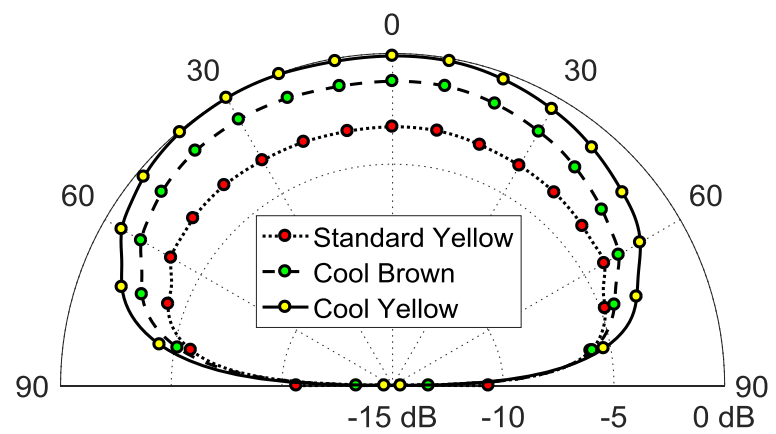

Fig.7. Measured scattering patterns of a normally incident infrared beam $(\lambda=940 \mathrm{~nm})$ on rough sheets of different materials and colors

\subsection{Wavelength Performance Dependence of the Indoor OWC System}

In indoor OWC systems, white Gaussian noise may be encountered due to the existence of ambient visible and invisible light within the room space. The signal strength expresses the quality of an infrared OW link under the encountered noise due to the ambient radiation found thorough the room area. In the present analysis, the signal strength is defined as the optical power density expressed in $\mathrm{nW} / \mathrm{cm}^{2}$. Fig. (8) is devoted to illustrate the dependence of the infrared signal strength and the corresponding SNR, at the central point on the room floor, on the operating wavelength for the three types of wall paint materials. Throughout this text, the parameter values are taken as: $R_{D}=0.225, L_{c}=2.4 \mathrm{~mm}$ $\mathrm{P}_{\mathrm{Tx}}=1 \mathrm{~W}, \mathrm{~N}_{0}=10^{-10} \mathrm{~W} / \mathrm{cm}^{2} / \mathrm{Hz}$. The highest level of the signal strength and, hence, the SNR (over the entire range of the wavelength under investigation) are achieved when the room ceil is coated with the cool yellow paint due to its high reflectivity. For $\lambda>1000 \mathrm{~nm}$, the cool brown paint results in higher values of both signal strength and the corresponding SNR than those obtained in the case of standard yellow paint. On the other hand, for $400<\lambda<1000 \mathrm{~nm}$, the signal strength as well as its SNR obtained when the room ceil is coated with a standard yellow paint exceeds those that are achieved when the cool brown paint is used.

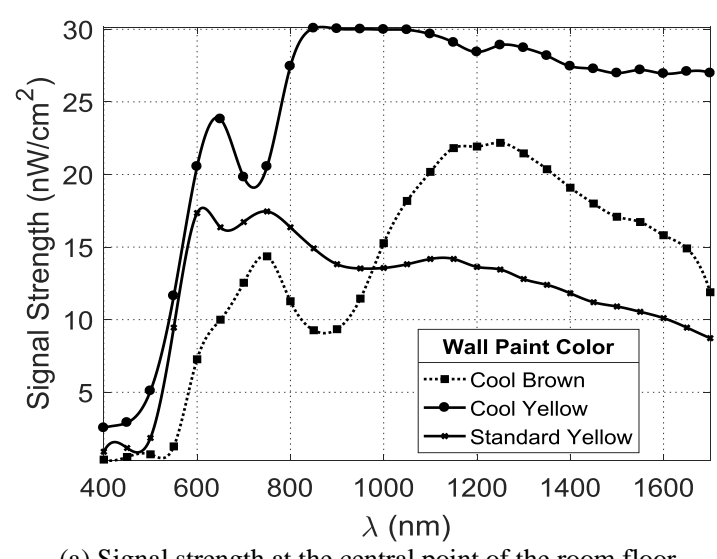

(a) Signal strength at the central point of the room floor

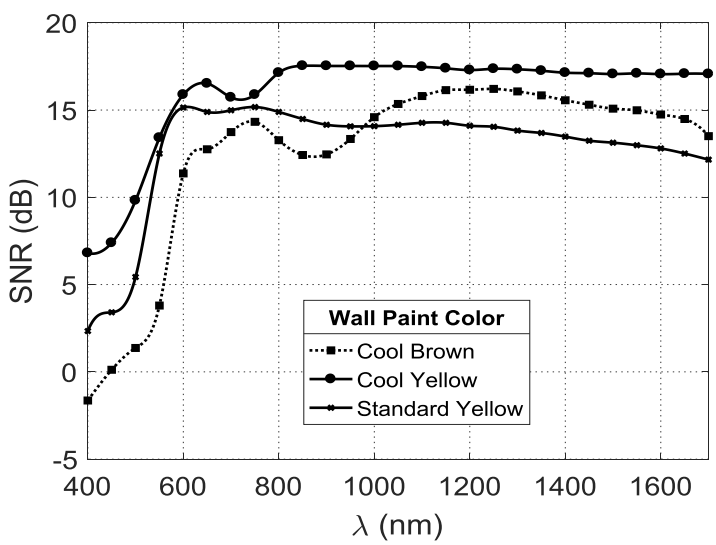

(b) SNR in single diffuse OW link

Fig.8. Wavelength dependence of the signal strength and the corresponding SNR in single diffuse OW link for the three types of the inner wall coating

In order to assess the performance of indoor OWC system, employing $M$-ary phase shift keying modulation technique, the dependence of the ratio of the data bit energy to the white noise power spectral density $\left(\mathrm{E}_{\mathrm{b}} / \mathrm{N}_{0}\right)$ is plotted, as a function of the wavelength, in Fig.(9-a). The corresponding BER is calculated and drawn, versus $\lambda$, in Fig.(9-b) for the underlined types of the wall paints. It is clear that the cool yellow paint has the lowest, relative to the considered three paint materials, BER. Within the wavelength range $800-1400 \mathrm{~nm}$, the cool yellow paint results in the best value of the BER which is less than $10^{-5}$ (for transmitted power of $1 \mathrm{~W}$ ). For the cool brown paint, on the other hand, the BER is less than $10^{-4}$ within the wavelength range $1100-1300 \mathrm{~nm}$. The standard yellow paint results in a BER below $10^{-3}$ within the wavelength range 550-1250nm. 


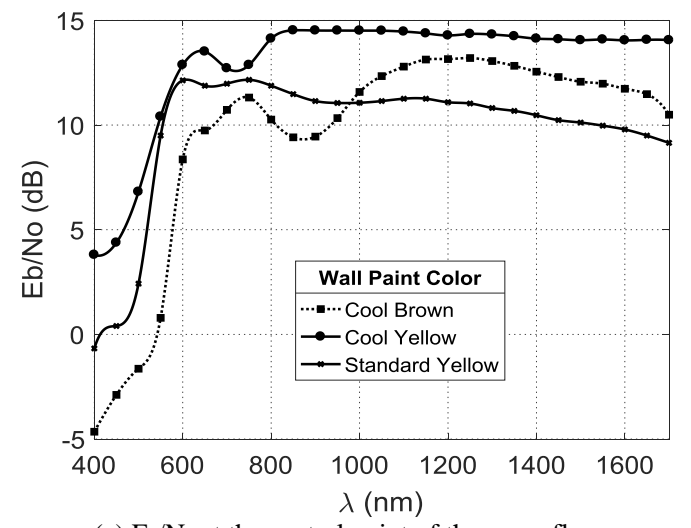

(a) $\mathrm{E}_{\mathrm{b}} / \mathrm{N}_{0}$ at the central point of the room floor

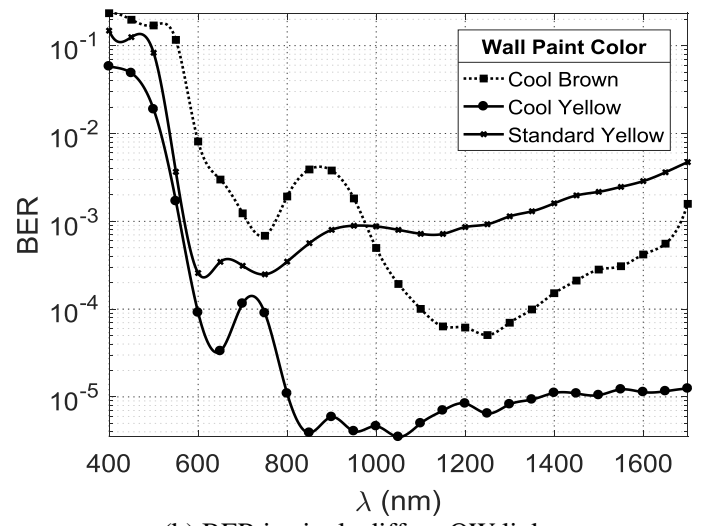

(b) BER in single diffuse OW link

Fig.9. Wavelength dependence of the ratio $\mathrm{E}_{\mathrm{b}} / \mathrm{N}_{0}$ and the corresponding BER, in single diffuse OW link, for the three types of inner wall coating

\subsection{Transmitted Power Performance characteristics of the Indoor OWC System}

\section{A. Visible-Light-Based OWC Link}

Here, we are interested in studying the effect of the transmitted optical power on the performance of a singlediffuse OW link operating in the visible range $(\lambda=550 \mathrm{~nm})$ of electromagnetic spectrum. To attain this objective, the signal strength at the central point of the room floor is evaluated and the result of this evaluation is plotted, versus the power of the primary optical beam for the three considered types of the inner wall coating, as Fig.(10-a) displays. As the signal strength is expressed in terms of the optical power intensity $\left(\mathrm{nW} / \mathrm{cm}^{2}\right)$, it is linearly increased with the transmitted power. On the other side, as the SNR is calculated in $\mathrm{dB}$, it exhibits a log-function dependence on the transmitted power as shown in Fig.(10-b). The displayed results of these plots demonstrate that the cool yellow paint gives the best signal strength and, hence, the best SNR for all the power levels. Also, it is clear that, within the spectrum of visible light, the signal strength and SNR obtained in the case where the room ceil is coated with a standard yellow paint are higher than those obtained when the cool brown paint is employed.

To assess the performance of indoor OWC system employing M-ary phase shift keying modulation scheme $(\mathrm{M}=8)$, the data bit energy to the power spectral density, of the white noise, ratio $\left(\mathrm{E}_{\mathrm{b}} / \mathrm{N}_{0}\right)$ is computed and traced, versus the power of the primary beam, for the tested three types of the inner wall coating, as Fig.(11-a) shows. The corresponding BER is evaluated and plotted, against the transmitted power, in Fig.(11-b) for the same types of the wall paints. It is clear that the BER decreases linearly with the transmitted power. Irrespective of the power level, the cool yellow paint presents the lowest, relative to the other under examination paint materials, BER. Additionally, it is noted that within the spectrum of the visible light, the standard yellow paint exhibits a BER which is significantly lower than that obtained in the case of the cool brown paint.

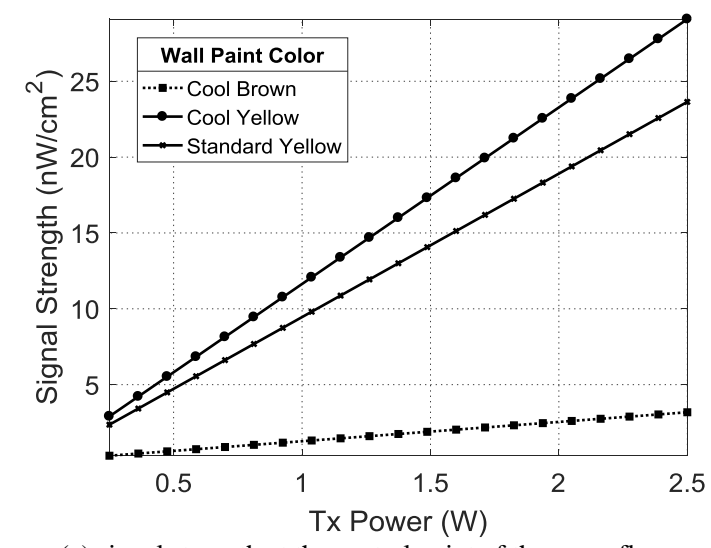

(a) signal strength at the central point of the room floor

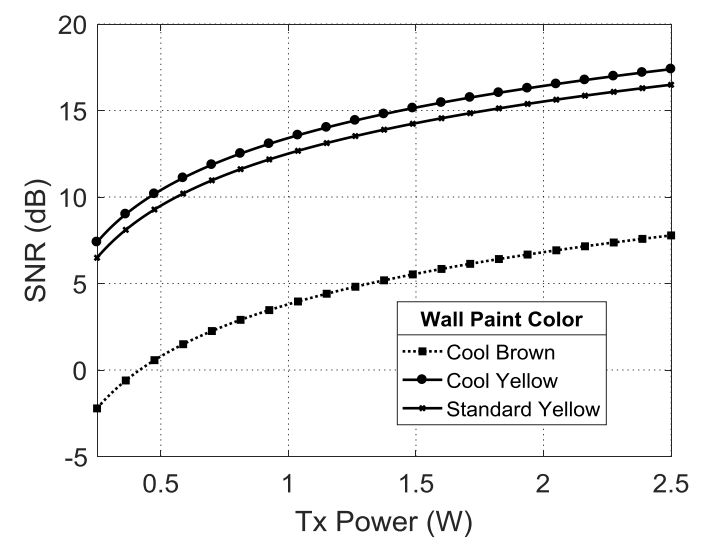

(b) SNR in single diffuse OW link

Fig.10. Power dependence of the signal strength and the corresponding SNR, in single diffuse OW link, on the power of the primary beam for the three types of the inner wall coating 


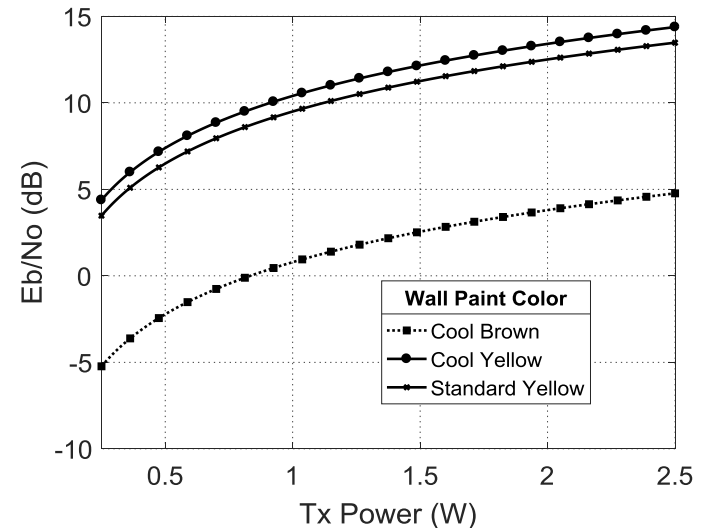

(a) $\mathrm{E}_{\mathrm{b}} / \mathrm{N}_{0}$ at the central point of the room floor

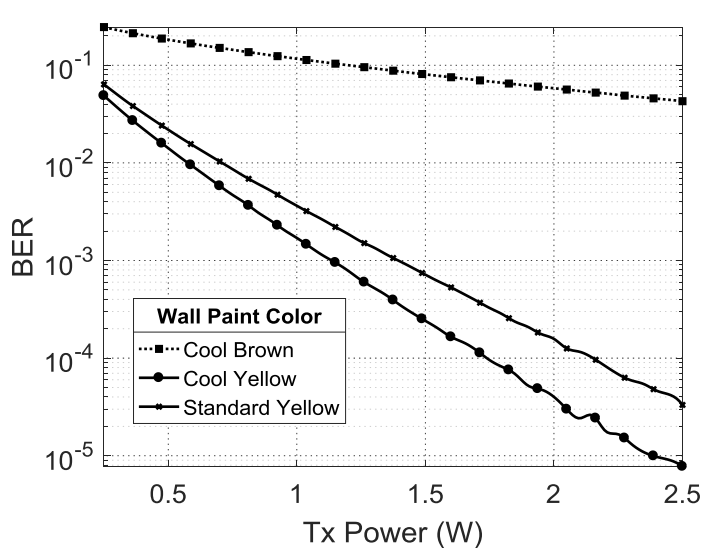

(b) BER in single diffuse OW link

Fig.11. Power dependence of the ratio $\mathrm{E}_{\mathrm{b}} / \mathrm{N}_{0}$ and the corresponding BER in single diffuse OW link on the power of the primary beam for the three types of the inner wall coating

\section{B. Infrared-Based OWC Link}

Here, we are going to investigate the performance, of single-diffuse OW link operating at $\lambda=1550 \mathrm{~nm}$, dependence on the power of the primary beam. Firstly, we calculate the signal strength, at the central point of the room floor, for numerous values of the transmitted power for each one of the three types of the inner wall coatings and the obtained results are plotted as visualized in Fig.(12-a). The behavior of the curves of this plot demonstrates that as the signal strength is expressed in terms of the optical power intensity $\left(\mathrm{nW} / \mathrm{cm}^{2}\right)$, it increases linearly, with a slope dependent on the light color, as a function of the transmitted power of the light beam. To show to what extent the background noise can affect the behavior of the system, the SNR, corresponding to each signal strength, is evaluated as Fig.(12-b) depicts. As the SNR is expressed in $\mathrm{dB}$, it exhibits a log-function dependence on the transmitted power. The displayed results show that the cool yellow paint yields the best signal strength and, consequently, the best SNR. Unlike the case of visible light, it is noted that, within the infrared spectrum, the signal strength and SNR obtained when the ceil of the room is coated with a cool brown paint are higher than those attained when the ceil is coated with the standard yellow paint.

For $M$-ary phase shift keying modulation scenario, the performance of the indoor OWC system can be assessed by calculating the ratio of the data bit energy to the power spectral density of the white noise $\left(\mathrm{E}_{\mathrm{b}} / \mathrm{N}_{0}\right)$ and plotting it as a function of the power of the primary beam for each one of the under examination types of the inner wall coating when $\lambda=1550 \mathrm{~nm}$. Fig.(13-a) illustrates the trace of the obtained results. Fig.(13-b) shows the corresponding BER as a function of the transmitted power for the same types of wall paints. It is observed that the BER decreases linearly with the transmitted power. Additionally, the cool yellow paint is the only one, amongst the considered wall paint materials, that gives the lowest BER regardless of the power level. It should be noted also that, within the spectrum of the infrared, the cool brown paint has a BER which is significantly lower than that achieved in the case of the standard yellow paint.

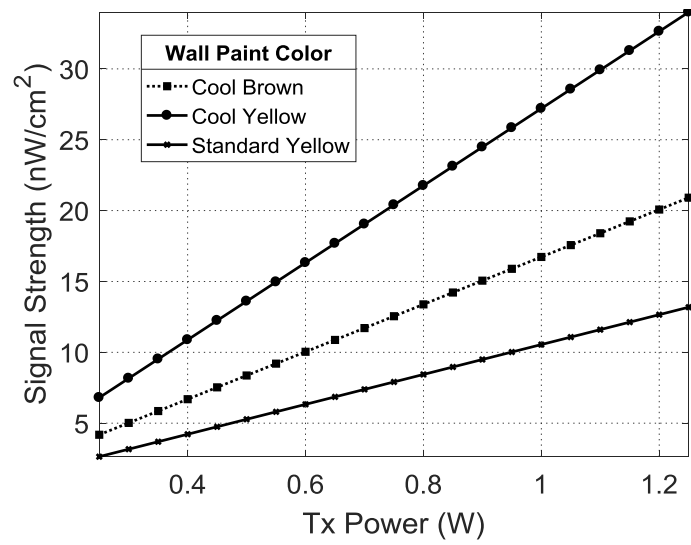

(a) Signal strength at the central point of the room floor

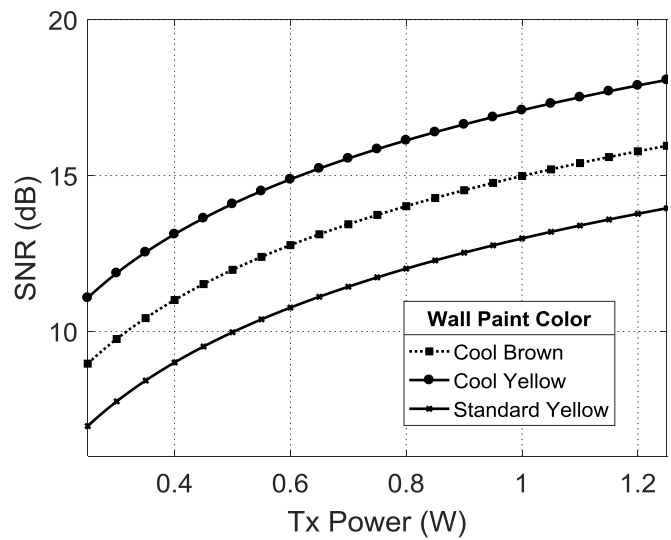

(b) SNR in single diffuse OW link

Fig.12. Power dependence of the signal strength and the corresponding SNR in single diffuse OW link on the power of the primary beam for the three types of the inner wall coating 


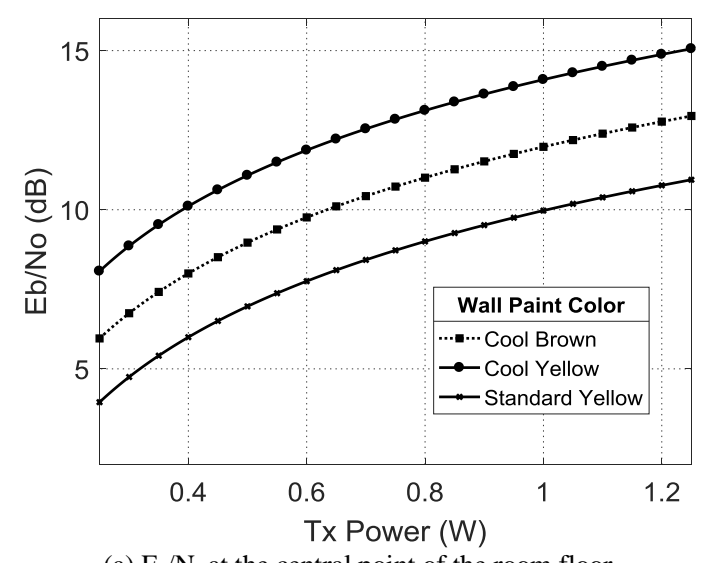

(a) $\mathrm{E}_{\mathrm{b}} / \mathrm{N}_{0}$ at the central point of the room floor

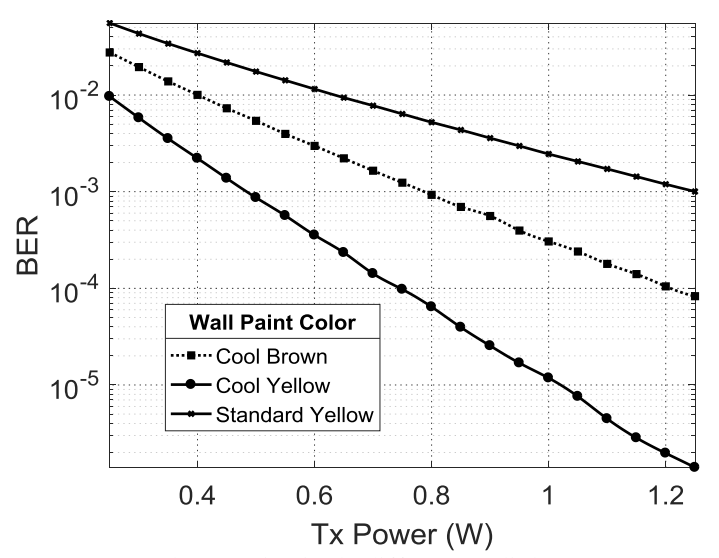

(b) BER in single diffuse OW link

Fig.13. Power dependence of the ratio $E_{b} / N_{0}$ and the corresponding BER in single diffuse OW link on the power of the primary beam for the three types of the inner wall coating

\section{Effect of Wall Coating on the Signal Coverage of Indoor OW Links}

In the diffuse OW link under consideration, it is assumed that the primary beam is normally incident on a Lambertian rough surface mounted at the center of the room ceil, as Fig. (1) reveals. The operating wavelength lies within the infrared spectrum $(\lambda=1000 \mathrm{~nm})$. The room is assumed to be of the same dimensions as previously taken and the Lambertian surface roughness has the same value as previously given in our practical measurements. Under these conditions, the distribution of the signal strength over the different locations within the cuboidal room is calculated for the three types of paint materials that may be considered as the coat of the Lambertian surface. It should be noted that the signal strength is expressed in terms of the optical power intensity $\left(\mathrm{nW} / \mathrm{cm}^{2}\right)$. Other operating parameters are of the same values as previously supposed and the bit rate is taken as $R_{b}=10 \mathrm{Mbps}$. The signal strength distribution is presented in Fig. (14) over the area of the room floor. Fig. (15) shows the corresponding distribution of the SNR over the floor area of the same room. Also, the distribution of the ratio $\mathrm{E}_{\mathrm{b}} / \mathrm{N}_{0}$, the BER and the symbol error rate (SER) over the same area are drawn in Figs. (16-18), respectively. It is clear that, for the three types of wall paint, the signal strength, the $\mathrm{SNR}$, and the ratio $\mathrm{E}_{\mathrm{b}} / \mathrm{N}_{0}$ have much lower values at the room corners than their values at the central area of the room. Consequently, the BER and the SER have much lower levels at the central area of the room than their levels at the room corners for the three types of wall paints. It should also be noted that the cool yellow paint gives the best performance regarding the level of the signal strength, the $\mathrm{SNR}$, and the ratio $\mathrm{E}_{\mathrm{b}} / \mathrm{N}_{0}$. As a consequence of this action, the cool yellow painting of the Lambertian surface gives the lowest BER and SER with respect to the other paint materials. At the operating wavelength $(\lambda=1000 \mathrm{~nm})$ cool brown paint donates the better values of the signal strength, SNR, the ratio $\mathrm{E}_{\mathrm{b}} / \mathrm{N}_{0}, \mathrm{BER}$, and the SER than those obtained with the standard yellow paint.

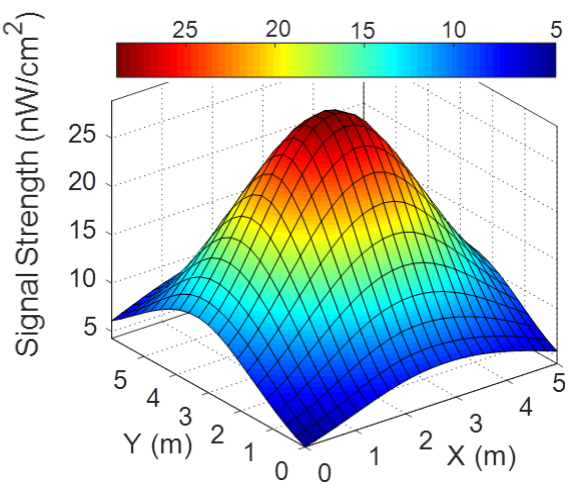

(a) Cool yellow paint

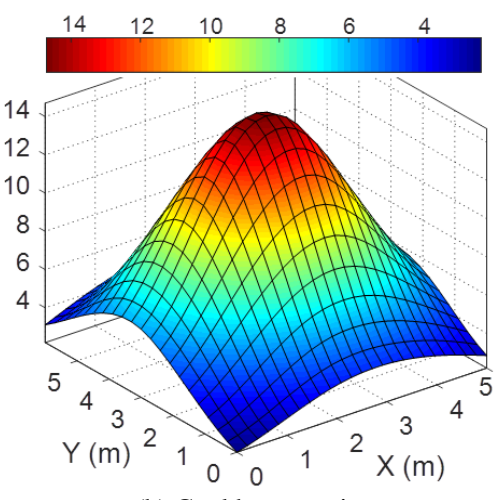

(b) Cool brown paint

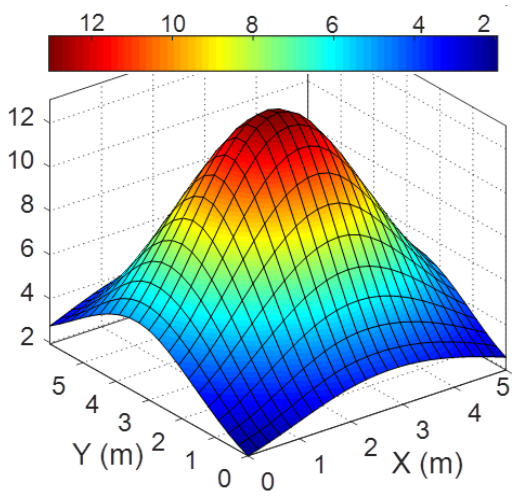

(c) Standard yellow paint

Fig.14. Signal strength distribution over the room floor area in the single-diffuse infrared OW link for different paint materials $\lambda=1000 \mathrm{~nm}$. 


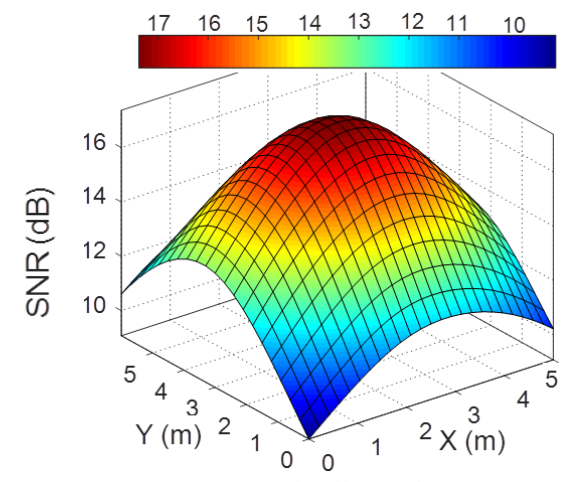

(a) Cool yellow paint

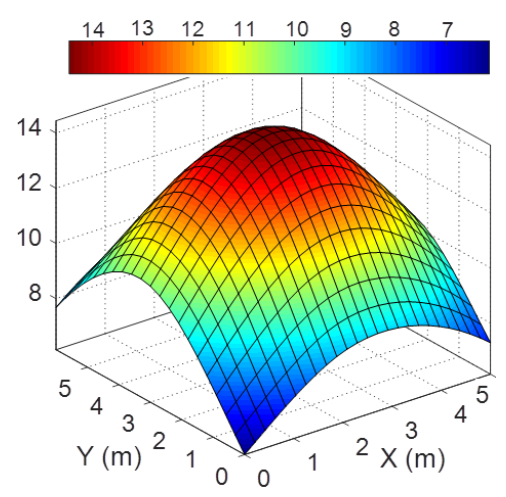

(b) Cool brown paint

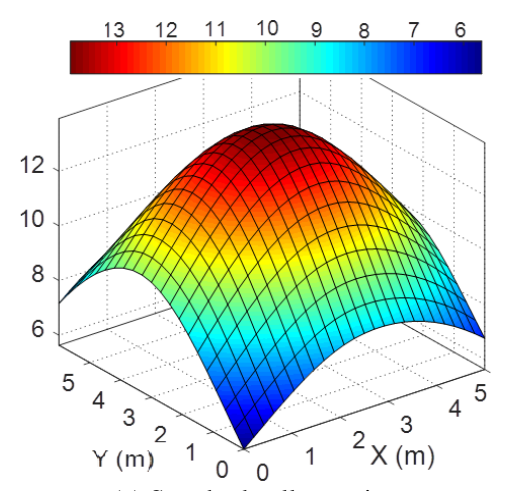

(c) Standard yellow paint

Fig.15. SNR distribution over the room floor area in single-diffuse infrared OW link for different paint materials $\lambda=1000 \mathrm{~nm}$.

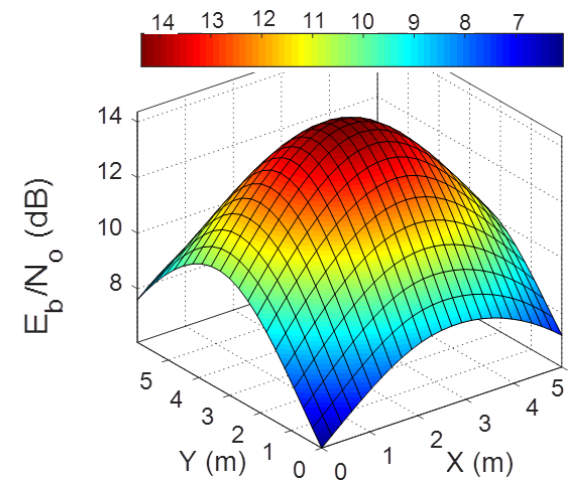

(a) Cool yellow paint

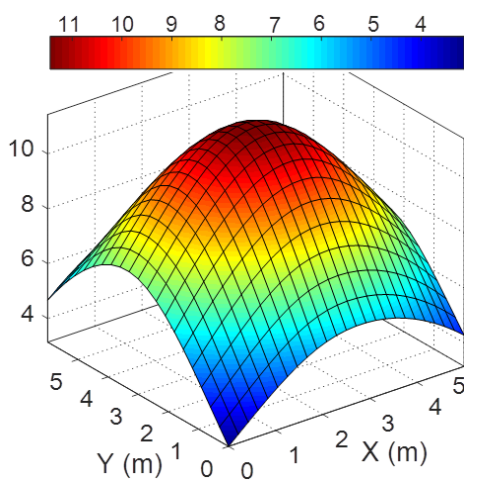

(b) Cool brown paint

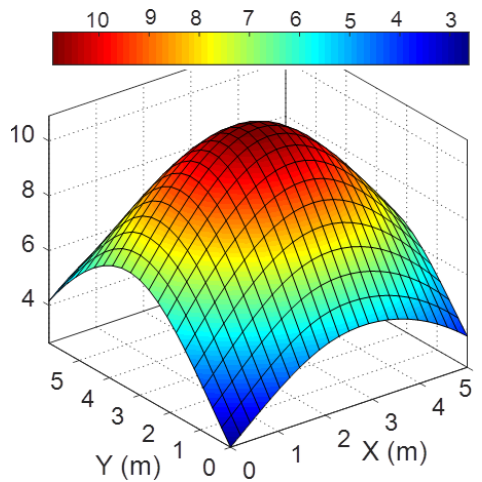

(c) Standard yellow paint

Fig.16. $\mathrm{E}_{b} / \mathrm{N}_{0}$ distribution over the room floor area in single-diffuse infrared OW link for different paint materials

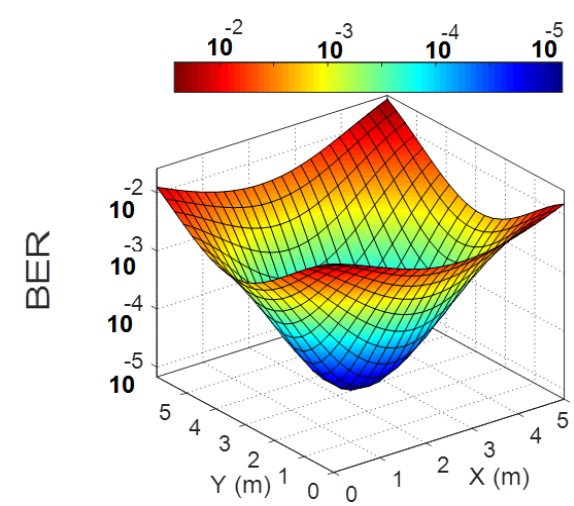

(a) Cool yellow paint

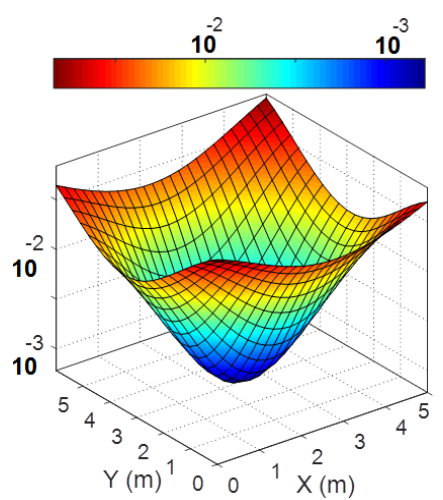

(b) Cool brown paint

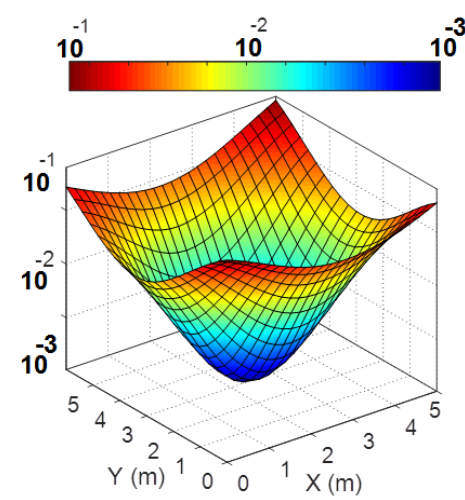

(c) Standard yellow paint

Fig.17. BER distribution over the room floor area in single-diffuse infrared OW link for different paint materials

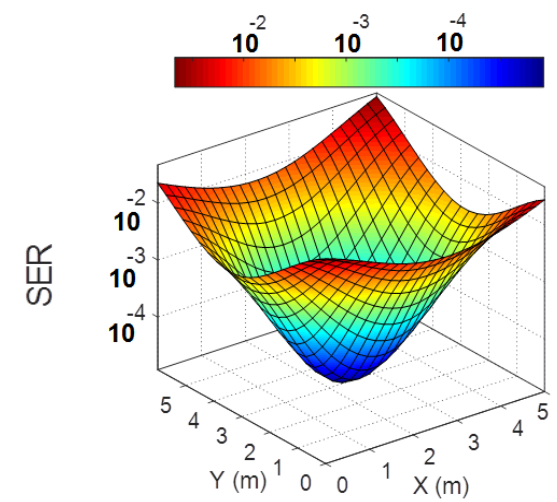

(a) Cool yellow paint

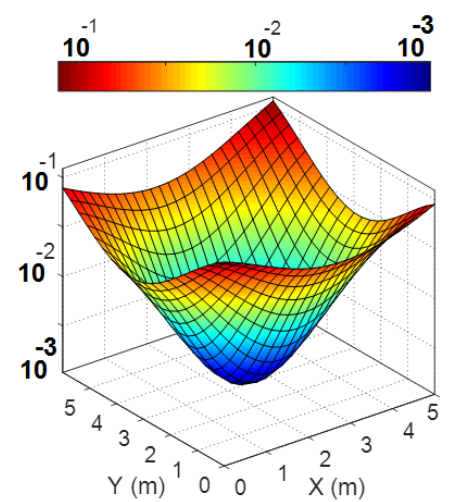

(b) Cool brown paint

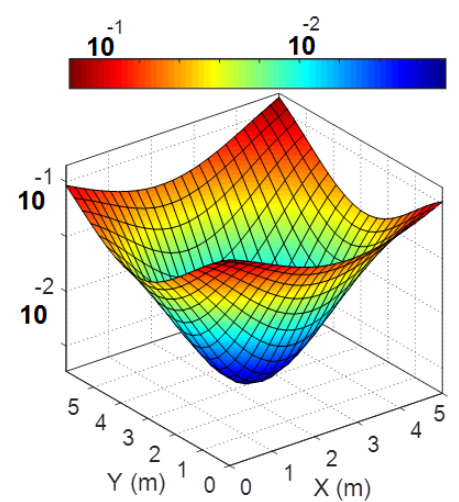

(c) Standard yellow paint

Fig.18. SER distribution over the room floor area in single-diffuse infrared OW link for different paint materials. 


\section{Conclusions}

This paper investigates the performance of the indoor OWC systems with single-diffuse topology. The GTD-RT procedure is proposed to evaluate the electromagnetic scattering from a rough surface of quite arbitrary statistical parameters. The algorithm developed in this research can be considered as a higher order GTD-RT that accounts for multiple bounces of an incident ray. The accuracy of the obtained results is verified through the comparison with the experimental measurements of the scattering pattern of a light beam incident on samples of rough sheets with specific statistical properties. The proposed GTD-RT strategy can be considered more accurate than the geometrical optics (GO) approximation and Kirchhoff approximation (KA) procedures and more computationally efficient than Monte Carlo averaging scenario.

The dependency of the indoor OWC system performance on the statistical properties of the Lambertian rough surface of the inner walls is analyzed. The optical signal strength distribution over the receiving area inside the room and the resulting BER due to unavoidable ambient light sources causing unwanted interference were evaluated and used as metrics for system performance goodness. Experimentally, it is shown that the infrared-reflective material of the wall coatings has a greater effect on the infrared reflectance than the lighter color of the standard materials. This means that a cool dark coating on the room walls has a higher infrared-reflectance than a conventional light coating, which in turn leads to increase the performance of the indoor OW links. Additionally, it is observed that the surface roughness of the matte painting on the Lambertian diffuse surface has a major effect on the indoor OWC system performance. Higher degree of roughness improves the BER at the central area of the room whereas it causes relatively higher BER at the corner points. Finally, it may be concluded that a matter of compromise is to select the optimum degree of roughness of the Lambertian diffuse surface to get high performance over the most active areas inside the room in OWC systems that implement single-diffuse topology.

\section{References}

[1] Heikki Koivo, Mohammed Elmusrati (2009). Systems Engineering in Wireless Communications. John Wiley \& Sons Ltd.

[2] IBM report (1995). An Introduction to Wireless Technology. International Technical Support Organization.

[3] Mohamed B. El-Mashade, Ehab A. Hegazy (2020). Performance Prediction of OFDM-Based Cognitive Radio for NextGeneration Networking Capabilities. International Journal of Recent Technology and Engineering (IJRTE), 9(1), 1911-1919.

[4] E.Coser, V.Moritz, A. Krenzinger and C.Ferreira (2015). Development of paints with infrared radiation reflective properties. Polímeros, 25(3), 305-310.

[5] G. Hugo (2001). Effects of low emissive wall coatings on thermal comfort and energy consumption. High Temperatures High Pressures, 33(1), 1-8.

[6] H. Akbari, P. Berdahl, R. Levinson, S. Wiel, B.Miller and A. Desjarlais (2006). Cool color roofing materials. CA: Lawrence Berkeley National Laboratory Berkeley.

[7] A. Libbra, L. Tarozzi, A. Muscio and M. Corticelli (2011). Spectral response data for development of cool coloured tile coverings. Optics \& Laser Technology, 43(2), 394-400.

[8] A. Krewinghaus (1969). Infrared reflectance of paints. Applied optics, 8(4), 807-812.

[9] V. Malshe, and A. Bendiganavale, (2008). Infrared reflective inorganic pigments. Recent Patents on Chemical Engineering, $1(1), 67-79$.

[10] T. Sowade (2011). IR-reflecting pigments. Technical Paper, Heubach, Germany, 1-4.

[11] A. Pisello, F. Cotana and L. Brinchi (2014). On a cool coating for roof clay tiles: development of the prototype and thermalenergy assessment. Energy procedia, No. 45, 453-462.

[12] A. Pisello, F. Cotana, A. Nicolini, and L. Brinchi (2013). Development of clay tile coatings for steep-sloped cool roofs. Energies, 6(8), 3637-3653.

[13] Pooja Kumari, Rajeev Thakur (2017). BER Evaluation of FSO Link with Hybrid Amplifier for Different Duty Cycles of RZ Pulse in Different Conditions of Rainfall. I.J. Wireless and Microwave Technologies, 1, 1-12.

[14] H. H. Qamar, K. F. A. Hussein, and M. B. El-Mashade (2019). Assessment of Signal Strength in Indoor Optical Wireless Communications Using Diffuse Infrared Radiation. 36th National Radio Science Conference (NRSC), IEEE.

[15] Shaela Sharmin, Shakil Mahmud Boby (2020). Characterization of WLAN System for $60 \mathrm{GHz}$ Residential Indoor Environment Based on Statistical Channel Modeling. I.J. Wireless and Microwave Technologies, 2, 42-58.

[16] M Mubasher Hassan, G M Rather (2020). Free Space Optics (FSO): A Promising Solution to First and Last Mile Connectivity (FLMC) in the Communication Networks. I.J. Wireless and Microwave Technologies, 4, 1-15.

[17] G.W. Marsh; J.M. Kahn (1994). 50-Mb/s diffuse infrared free-space link using on-off keying with decision-feedback equalization. IEEE Photonics Technology Letters, 6(10),1268-1270.

[18] J.M. Kahn; W.J. Krause; J.B. Carruthers (1995). Experimental characterization of non-directed indoor infrared channels. IEEE Transactions on Communications, 43(2), 1613-1623.

[19] A.G. Al-Ghamdi, J.M.H. Elmirghani (2004). Analysis of diffuse optical wireless channels employing spot-diffusing techniques, diversity receivers, and combining schemes. IEEE Transactions on Communications, 52(10), 1622-1631. 


\section{Authors' Profiles}

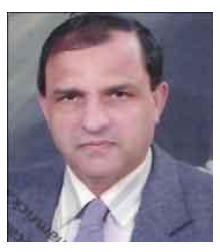

Mohamed Bakry El-Mashade received the B.Sc. degree in electrical engineering from Al-Azhar University, Cairo, in 1978, the M.Sc. degree in the theory of communications from Cairo University, in 1982, Le D.E.A d'Electronique (Spécialité: Traitment du Signal), and Le Diplôme de Doctorat (Spécialité: Composants, Signaux et Systems) in optical communications, from USTL, L'Academie de Montpellier, Montpellier, France, in 1985 and 1987, respectively. He serves on the Editorial Board of several International Journals. He has also served as a reviewer for many international journals. He was the author of more than 60 peer-reviewed journal articles and the coauthor of more than 60 journal technical papers as well as three international book chapters. He received the best research paper award from International Journal of Semiconductor Science \& Technology in 2014 for his work on "Noise Modeling Circuit of Quantum Structure Type of Infrared Photodetectors". He won the Egyptian Encouraging Award, in Engineering Science, two times (1998 and 2004). He was included in the American Society 'Marquis Who's Who' as a 'Distinguishable Scientist' in 2004 and in the International Biographical Centre of Cambridge (England) as an 'Outstanding Scientist' in 2005. He has been

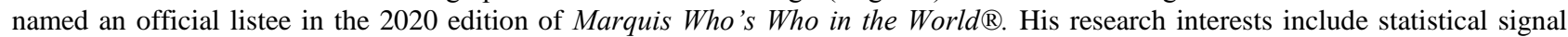
processing, digital and optical signal processing, free space optical communications, fiber Bragg grating, quantum structure family of optical devices, SDR, cognitive radio, and software defined radar \& SAR.

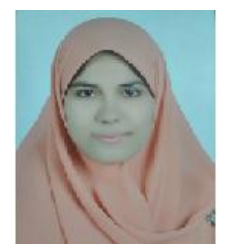

Hanna Helmy Qamar was born in 1992. She received her Bachelor's Degree, with Honor's, in Electronics and Communications Engineering from Al-Azhar University, Egypt, in 2015. She received her Master's Degree in optical communications from Al-Azhar University in 2020, Egypt. Her area of interest is free space optical communications.

How to cite this paper: Mohamed B. El-Mashade, Hanaa H. Qamer, " Impact of Wall Coating on the Behavior of Indoor OWC under Diffuse Topology", International Journal of Wireless and Microwave Technologies(IJWMT), Vol.11, No.1, pp. 1-14, 2021.DOI: 10.5815/ijwmt.2021.01.01 